\title{
A collective investment problem in a stochastic volatility environment: The impact of sharing rules
}

\author{
An Chen ${ }^{1}$ (ID) Thai Nguyen ${ }^{2} \cdot$ Manuel Rach $^{1}$ \\ Accepted: 4 February 2021 / Published online: 8 March 2021 \\ (c) The Author(s) 2021
}

\begin{abstract}
It is typical in collectively administered pension funds that employees delegate fund managers to invest their contributions. In addition, many pension funds still need to sustain guarantees (prescribed by law) in spite of the current low interest environment. In this paper, we consider an optimal collective investment problem for a pool of investors who (implicitly) demand minimum guarantees by deriving utility from the wealth exceeding their guarantees in two financial market settings, one with a stochastic and one with a constant volatility. We find that individual investors' well-being will not be worsened through the collective investment in both financial markets, as individual optimal solutions are attainable if a financially fair statedependent sharing rule is applied. When more prevailing sharing rules like linear rules are applied, this holds no longer. Furthermore, the degree of sub-optimality imposed by linear sharing rules is more pronounced in the stochastic volatility market than in the constant volatility market.
\end{abstract}

Keywords Collective investment problems $\cdot$ Stochastic volatility $\cdot$ portfolio insurance $\cdot$ Sharing rules

JEL Classification G11 · G23

\section{Introduction}

There exist various reasons for fund delegation in today's world, one of the most prominent examples being a collectively administered pension fund. There are two main types of occupational pensions schemes: In a defined benefit (DB) scheme, the sponsoring com-

\section{An Chen}

an.chen@uni-ulm.de

Thai Nguyen

thai.nguyen@act.ulaval.ca

Manuel Rach

manuel.rach@uni-ulm.de

1 Institute of Insurance Science, Ulm University, Helmholtzstrasse 20, 89069 Ulm, Germany

2 École d'Actuariat, Université Laval, 2425 rue de 1'Agriculture, Québec, Québec G1V 0A6, Canada 
panies promise their employees a guaranteed pension payment. In a defined contribution (DC) scheme, the benefit at retirement depends on the performance of the investment returns experienced during the plan membership. Consequently, in a DC scheme, the market risk is carried completely by the employees instead of the employers. ${ }^{1}$ Very recently, people have started to believe that hybrid pension plans combining the DC and DB plan might meet the requirements of employees and employers even better. A key component of such hybrid schemes is to provide safety by offering a minimum guarantee (which is lower than in pure DB schemes), and, simultaneously, to let employees participate in potential upside scenarios of the markets. ${ }^{2}$ In such pensions, fund managers shall take account of the guarantee requirement in their portfolio planning, while simultaneously capturing the members' risk preferences in the investment strategy to provide acceptable bonuses to the members in wellperforming markets. Another reason for fund delegation, besides collectively administered pension funds, is explained, for example, in Kim et al. (2016): Many households display investment inertia because handling investments costs time and energy. The authors find that delegation can be beneficial for individual investors.

In this article, we consider an optimal investment problem of a fund manager who invests on behalf of a collective of individuals requiring a minimum guaranteed payment in a stochastic volatility framework. In a utility maximization framework, it is common in the literature to assume that individuals implicitly satisfy their guarantee requirements by deriving utility only from the residual wealth exceeding the guarantee, ${ }^{3}$ see, for example, Basak (2002), Balder and Mahayni (2010) and Zieling et al. (2014). ${ }^{4}$ Each of the individuals in the collective may demand a certain guarantee. We allow individuals with various degrees of risk aversion to choose a different guarantee level. The utility function used by the fund manager is itself defined by an optimization problem in such a way that the weighted sum of the individual utility functions is maximized for a given vector of positive weights. Due to the inclusion of guarantee requirements, this is a generalization of a popular utility funciton (with no minimum subsistence level) in the literature [see, for example, Dumas (1989), Karatzas et al. (1990), Xia (2004), Pazdera et al. (2016), Branger et al. (2018b) and Chen et al. (2021)]. The fund manager then sets up a collective investment strategy such that all these individual guarantees are met. We not only consider the Black-Scholes setting with a constant volatility, but also move beyond normally distributed returns and describe the evolution of the stock with a more general stochastic volatility model in the sense of Heston (1993). A stochastic volatility model is more realistic than a model with constant volatility, for it allows to explain stylized facts often observed in financial markets such as heavy tails, volatility clustering, and the smile of implied volatilities [see Cont and Tankov (2004)]. In such a stochastic volatility model, which leads to bigger tail risks, appropriate fund management under portfolio

\footnotetext{
1 For further details on DB and DC schemes, see also OECD (2018).

2 An overview over existing hybrid schemes can, for example, be found in Turner (2014). A literature review on dynamic hybrid pension products is provided by Hambardzumyan and Korn (2019).

3 Note that, in a Black-Scholes setting, the resulting optimal investment strategy for an individual investor with such utility preferences is a so-called constant proportion portfolio insurance (CPPI) strategy, a rather popular type of portfolio insurance strategies [see, for example, Black and Jones (1987), Black and Perold (1992), Basak (2002) and, more recently, Temocin et al. (2018), and for the relevance of CPPI strategies in practice see Pain and Rand (2008)]. While this type of strategy is optimal for a single individual with risk preferences as described above, we find that this result holds no longer for a collective of individuals who jointly invest their initial wealth. Further details regarding this result and CPPI strategies are provided in Sect. 3.2 of this article.

4 While we follow this first approach in this article, a second popular approach would be to impose a minimum guarantee constraint in the utility maximization problem, as done, for example, in Jensen and Sørensen (2001) and Hambardzumyan and Korn (2019).
} 
insurance becomes even more important than in a model with normally distributed returns, because the probability of extremal market scenarios increases [see Chen et al. (2018)]. In this article, we are particularly interested in finding out the influence of such a more realistic financial market modeling on the expected utility of the individual investors, and comparing it to the constant volatility framework.

We show that, under both constant and stochastic volatility, individual optimal solutions are achievable if a state-dependent sharing rule is applied to the optimal collective terminal wealth and the financial fairness condition in the sense of Bühlmann and Jewell (1979) and Schumacher (2018) is imposed. In other words, individual welfare does not deteriorate in both financial markets if an appropriate state-dependent sharing rule is applied. Under more prevailing sharing rules, like linear ones, this result holds no longer, as linear sharing rules impose a certain suboptimality to the collective [see, for example, Jensen and Nielsen (2016)]. Then, either all the individuals in the collective suffer a loss or an unfair distribution of the terminal wealth, where some individuals benefit at the cost of others, results. To assess the losses imposed by linear sharing rules in both financial markets, we compare the statedependent sharing rule to two linear sharing rules: one satisfying the financial fairness and one not. If the linear sharing rule does not fulfill the fairness condition, some individuals in the collective are better off, but the majority of investors is largely worse off than in the individual optimization problem. When a financially fair linear sharing rule is applied, all the individuals suffer a (relatively) small loss. In this sense, a financially fair linear sharing rule performs better from a fund manager's point of view who wants to consider all the individuals in the collective in a fair way. A comparison between the constant and stochastic volatility framework reveals that the degree of sub-optimality imposed by linear sharing rules is larger under stochastic volatility.

Individuals' utility optimization in incomplete stochastic volatility markets has been considered extensively in the literature [see, for example, Pham (2002), Fleming and Hernández-Hernández (2003), Chacko and Viceira (2005), Kraft (2005) and Liu (2006)]. For common utility functions (for example power utility), the solution is available in closed form by applying a separation technique in the Hamilton-Jacobi-Bellman (HJB) equation resulting from the dynamic programming principle. Unfortunately, such a separation technique seems impossible in our collective utility maximization framework. Consequently, we rely on another way of solving the optimal investment problem: We complete the financial market using derivatives. This approach is well-documented in the literature and applied, for instance, in Liu and Pan (2003), Branger et al. (2008, 2017), Escobar et al. (2018) and Chen et al. (2018). Following this approach, we can determine the optimal terminal wealth levels and the dynamic trading strategies explicitly for our collective utility maximization problem in the stochastic volatility framework using the static martingale approach [see, for example, Cox and Huang (1989)]. Solving the collective optimization problem under a constant volatility is less complicated, as the constant volatility market is complete without adding derivatives. In this sense, our article contributes to the literature on utility maximization in incomplete stochastic volatility markets by the consideration of a collective utility maximization problem.

The remainder of the paper is organized in the following way: Sect. 2 introduces the utility preferences assumed for the individuals in the collective and, particularly, the collective utility function used for modeling the fund manager's preferences. Section 3 briefly presents the solution to the optimal collective investment problem in a constant volatility framework. Section 4 deals with the collective optimization problem in the Heston model which allows for stochastic volatility. In Sect. 5, we show that individual optimal solutions can be achieved through the collective investment under financial fairness and a state-dependent sharing rule. 
In Sect. 6, we discuss different sharing rules and compare the well-being of the investors in the collective under constant and stochastic volatility. Section 7 concludes the article and is followed by the appendix with one proof.

\section{Risk preferences}

In this section, we describe the basic assumptions regarding the preferences of the individuals. To model individual preferences, we mainly take account of the fact that the individuals are interested in obtaining a minimum payment and building their utility on the (residual) wealth exceeding the minimal guarantee.

\subsection{Individual preferences}

We consider a collective of $n$ individuals on a financial market. Each of the investors assigns her initial wealth $x_{i}$ to the fund manager for investment in financial assets at time 0 . We use a special type of HARA utility function of the form $U_{i, G^{i}}(v)=\frac{\left(v-G^{i}\right)^{1-\gamma_{i}}}{1-\gamma_{i}}$ with $\gamma_{i} \neq 1$, $\gamma_{i}>0$ for $i=1, \ldots, n$ to model each investor's preferences, where $G^{i}$ is investor $i$ 's subsistence level. This subsistence level will be referred to as a minimum guarantee that investor $i$ is interested in achieving from now on. The corresponding relative risk aversion is given by $\frac{\gamma_{i} v}{v-G^{i}}$ which is increasing in $G^{i}$ and $\gamma_{i}$. In the special case that $G^{i}=0$, we obtain constant relative risk aversion (CRRA) utility functions. Note that each investor derives her utility only from the difference between the total terminal wealth and the guarantee. This preference representation for individuals interested in sustaining a minimum guaranteed income is common in the literature, see, for example, Basak (2002), Balder and Mahayni (2010) and Zieling et al. (2014). The resulting inverse marginal utility function is denoted by

$$
I_{i, G^{i}}(\cdot):=\left(U_{i, G^{i}}^{\prime}\right)^{-1}(\cdot)=G^{i}+(\cdot)^{-\frac{1}{\gamma_{i}}} .
$$

\subsection{Collective utility function}

From now on, we assume that the $n$ investors delegate a fund manager to collectively invest their total initial wealth $x=\sum_{i=1}^{n} x_{i}$ on their behalf. Reasons for fund delegation can be different: For example, in an occupational pension context, it is common that beneficiaries do not administrate their contributions themselves. Instead, contributions are collectively managed by a pension fund manager. Another reason for fund delegation could be professional skills and knowledge of the fund manager, leading individual investors to believe that investment delegation is more beneficial for them than handling investments on their own. Further, Kim et al. (2016) observe that many individuals display investment inertia as managing money costs time and energy and show that delegation is valuable.

We assume that the fund manager does not charge any additional fees, so the total wealth $x$ is completely invested in financial assets. The fund manager's primal goal is to provide individual guarantees, as it is the case, for example, in many occupational pension schemes that are not of the pure DC type. ${ }^{5}$ In many other real-life fund delegation situations, fund managers might be more interested in maximizing their own compensations from advising

5 For instance, in all German pension schemes, some sort of guarantee had been prescribed until very recently in 2018 when a new pension scheme was introduced along with the "Betriebsrentenstärkungsgesetz". 
individual investors regarding the suitability of financial products. As in Kim et al. (2016), we assume that the fund manager behaves more on behalf of individual investors, that is, the fund manager's utility function reflects the individuals' utility and, more importantly, the fund manager aims to meet individual guarantees. We denote by $G$ the time- $T$-value of the collective guarantee which the fund manager needs to meet. Unless stated otherwise, we will always assume that this guarantee is equal to the sum of the individual guarantees, that is, $G=\sum_{i=1}^{n} G^{i}$. Additionally, we want to emphasize that the case with no guarantee is included throughout this article, as all the individual guarantees can always be set equal to zero.

Concerning the collective utility function (which the fund manager uses), we are inspired, for example, by Dumas (1989), Karatzas et al. (1990), Xia (2004), Pazdera et al. (2016), Branger et al. (2018b) and Chen et al. (2021). We assume that the fund manager uses the following (collective) utility function which depends on the collective and individual guarantees:

$$
U_{B, G}:(G, \infty) \rightarrow(0, \infty), \quad v \mapsto U_{B, G}(v)=\max _{\substack{v_{1} \geq G^{1}, \ldots, v_{n} \geq G^{n} \\ v=\sum_{i=1}^{n} v_{i}}} \sum_{i=1}^{n} \beta_{i} U_{i, G^{i}}\left(v_{i}\right),
$$

where $B=\left(\beta_{1}, \ldots, \beta_{n}\right)$ is a vector consisting of strictly positive numbers adding up to 1 . The vector $B$ controls how each individual investor is weighted in the collective investment problem. Note that the utility of the fund manager is only defined for values exceeding the collective guarantee. Lemma 1 states that $u_{B, G}$ is, in fact, a utility function, as it has already been shown in Branger et al. (2018b) for the case where all the individual guarantees are equal to zero.

Lemma $1 U_{B, G}$ is a strictly increasing and concave function on $(G, \infty)$ for all $G=\sum_{i=1}^{n} G^{i}$ with $G^{i} \geq 0$, whose inverse marginal utility is given by

$$
I_{B, G}(\cdot):=\left(U_{B, G}^{\prime}\right)^{-1}(\cdot)=\sum_{i=1}^{n} I_{i, G^{i}}\left(\frac{\cdot}{\beta_{i}}\right) .
$$

Proof The collective utility function given in (1) is itself defined through an optimization problem whose Lagrangian is given by

$$
\mathcal{L}=\sum_{i=1}^{n} \beta_{i} U_{i, G^{i}}\left(v_{i}\right)+y\left(v-\sum_{i=1}^{n} v_{i}\right) .
$$

The first order conditions are

$$
\frac{\partial \mathcal{L}}{\partial v_{i}}=\beta_{i} U_{i, G^{i}}^{\prime}\left(v_{i}\right)-y=0 \quad \Leftrightarrow \quad v_{i}=I_{i, G^{i}}\left(\frac{y}{\beta_{i}}\right) \quad \text { for all } i=1, \ldots, n .
$$

This results in

$$
v=\sum_{i=1}^{n} I_{i, G^{i}}\left(\frac{y}{\beta_{i}}\right)
$$

Now we define the function

$$
I_{B, G}:(0, \infty) \rightarrow(G, \infty), \quad y \mapsto I_{B, G}(y)=\sum_{i=1}^{n} I_{i, G^{i}}\left(\frac{y}{\beta_{i}}\right)
$$


Note that this function is strictly decreasing on $(0, \infty)$, that $\lim _{y \rightarrow 0} I_{B, G}(y)=\infty$ and that $\lim _{y \rightarrow \infty} I_{B, G}(y)=G$. Hence, for any $v \in(G, \infty)$ there exists a unique value $y \in(0, \infty)$ such that $I_{B, G}(y)=v$ for which the optimization problem (1) attains its maximum at $v_{i}=I_{i, G^{i}}\left(\frac{y}{\beta_{i}}\right)$. This maximum collective utility level is given by

$$
U_{B, G}(v)=\sum_{j=1}^{n} \beta_{j} U_{j, G^{j}}\left(v_{j}\right)=\sum_{j=1}^{n} \beta_{j} U_{j, G^{j}}\left(I_{j, G^{j}}\left(\frac{y}{\beta_{j}}\right)\right) .
$$

The first order derivative of $U_{B, G}$ can now be determined as

$U_{B, G}^{\prime}(v)=\sum_{j=1}^{n} \beta_{j} U_{j, G^{j}}^{\prime}\left(I_{j, G^{j}}\left(\frac{y}{\beta_{i}}\right)\right) I_{j, G^{j}}^{\prime}\left(\frac{y}{\beta_{j}}\right) \frac{d y}{\beta_{j} d v}=y \sum_{j=1}^{n} I_{j, G^{j}}^{\prime}\left(\frac{y}{\beta_{j}}\right) \frac{d y}{\beta_{j} d v}=y$,

where the last equality can be obtained from taking the derivative with respect to $v$ on both sides of (3). This leads to

$$
\left(U_{B, G}^{\prime}\right)^{-1}(y)=v=\sum_{i=1}^{n} I_{i, G^{i}}\left(\frac{y}{\beta_{i}}\right),
$$

which completes the proof.

\section{Constant volatility model}

We start our analysis by a brief consideration of the classic Black-Scholes model which assumes a constant volatility of the risky asset. It will serve as a comparison basis to the stochastic volatility case specified in Sect. 4.

\subsection{Financial market}

We consider a financial market consisting of a risk-free asset $B$ and a risky asset $S$. The risk-free asset $B$ is assumed to earn a constant interest rate $r$, that is,

$$
d B_{t}=r B_{t} d t, \quad B_{0}=1 .
$$

Let $\left\{W_{t}\right\}_{t \in[0, T]}$ be a standard Brownian motion on a probability space $(\Omega, \mathcal{F}, \mathbb{P})$ satisfying the usual hypothesis. The risky asset $S$ follows a geometric Brownian motion

$$
d S_{t}=\mu S_{t} d t+\sigma S_{t} d W_{t}, S_{0}=s .
$$

Here we assume that $\mu-r>0$ and $\sigma>0$. In this complete market, the state price density process is uniquely determined by the following stochastic differential equation:

$$
d \xi_{t}=-\xi_{t}\left(r d t+\chi d W_{t}\right), \quad \xi_{0}=1, \quad \chi=\frac{\mu-r}{\sigma} .
$$

The value $\xi_{t}$ can be interpreted as the state of the economy at time $t$ : The better the market performs, the lower $\xi_{t}$ gets. In the following sections, we will use this property to analyze the performance of the (state-dependent) terminal wealth and investment strategy under different market states. From now on, let $G_{t}^{i}=e^{-r(T-t)} G^{i}$ denote the time- $t$-value of the fixed level of guarantee investor $i$ requires, where $G^{i}=G_{T}^{i} \in\left(0, x_{i} e^{r T}\right)$. We assume an upper bound for the guarantee to ensure the feasibility of our optimization problems. 
If we denote by $\left\{\pi_{t}\right\}_{t \in[0, T]}$ the fraction of total wealth which is invested in the risky asset by the fund manager and assuming a self-financing trading strategy, the dynamics of the total wealth $\left\{X_{t}\right\}_{t \in[0, T]}$ are described by the following stochastic differential equation:

$$
d X_{t}=\left(r+\pi_{t}(\mu-r)\right) X_{t} d t+\sigma \pi_{t} X_{t} d W_{t}, \quad X_{0}=x .
$$

The trading strategy $\left\{\pi_{t}\right\}_{t \in[0, T]}$ is chosen from the following set of admissible strategies:

$$
\begin{aligned}
\mathcal{A}(x):= & \left\{\left\{\pi_{t}\right\}_{t \in[0, T]} \mid X_{0}=x,\left\{\pi_{t}\right\}_{t \in[0, T]}\right. \text { is progressively measurable, } \\
& \left.X_{t} \geq 0 \text { for all } t \geq 0, \int_{0}^{T} \pi_{s}^{2} d s<\infty \text { a.s. }\right\}
\end{aligned}
$$

\subsection{Collective optimization problem}

In a constant volatility framework, the collective optimization problem can be written down as

$$
\max _{\left(\pi_{t}\right)_{t \in[0, T]}} \mathbb{E}\left[U_{B, G}\left(X_{T}\right)\right] \text { subject to (6). }
$$

Due to the market completeness, this problem can be solved using the static martingale approach (Cox and Huang 1989), that is, by solving the static optimization problem

$$
\max _{X_{T}} \mathbb{E}\left[U_{B, G}\left(X_{T}\right)\right] \quad \text { subject to } \mathbb{E}\left[\xi_{T} X_{T}\right]=x
$$

for the optimal terminal wealth $X_{T}$ and then determining the optimal trading strategy from the optimal wealth. To ensure the feasibility of the optimization problem (8), we shall examine the following two integrability conditions:

$$
\begin{aligned}
\mathbb{E}\left[\xi_{T} I_{B, G}\left(\lambda \xi_{T}\right)\right] & <\infty, \\
\mathbb{E}\left[U_{B, G}\left(I_{B, G}\left(\lambda \xi_{T}\right)\right)\right] & <\infty,
\end{aligned}
$$

for all $\lambda>0$. Condition (9) ensures that the initial market value of the terminal wealth is finite for all possible values of the Lagrangian multiplier. Condition (10) ensures that the value function is finite for all possible values of the Lagrangian multiplier. Note that both conditions are fulfilled in our Black-Scholes financial market with (modified) power utility functions.

Due to the nice property of the collective utility function, particularly the explicit representation of the inverse marginal utility of $U_{B, G}$, we obtain the solution of the optimization problem (8) as

$$
X_{T}^{*}=I_{B, G}\left(\lambda \xi_{T}\right)=\sum_{i=1}^{n} I_{i, G^{i}}\left(\frac{\lambda}{\beta_{i}} \xi_{T}\right)=\sum_{i=1}^{n}\left(G^{i}+\left(\frac{\lambda}{\beta_{i}} \xi_{T}\right)^{-\frac{1}{\gamma_{i}}}\right),
$$

where $\lambda$ is the Lagrangian multiplier which can be uniquely determined from the budget constraint $\mathbb{E}\left[\xi_{T} X_{T}^{*}\right]=x$.

Remark 1 For $n=1$, Problem (8) is reduced to the individual optimization problem (taking individual $i$ with an initial wealth $x_{i}>G^{i} e^{-r T}$ as an example):

$$
\max _{X_{T}^{i}} \mathbb{E}\left[U_{i, G_{i}}\left(X_{T}^{i}\right)\right] \text { subject to } \mathbb{E}\left[\xi_{T} X_{T}^{i}\right]=x_{i} .
$$


The individual optimal solution for individual $\mathrm{i}$ is given by

$$
X_{T}^{(i, *)}=I_{i, G^{i}}\left(\lambda_{i} \xi_{T}\right),
$$

where $\lambda_{i}$ can be determined explicitly from the budget constraint $\mathbb{E}\left[\xi_{T} X_{T}^{(i, *)}\right]=x_{i}$.

From Eq. (11), we can derive the optimal wealth for any $t \in[0, T)$ as follows:

$$
\begin{aligned}
X_{t}^{*} & =\mathbb{E}\left[\frac{\xi_{T}}{\xi_{t}} X_{T}^{*} \mid \mathcal{F}_{t}\right] \\
& =G_{t}+\mathbb{E}\left[\frac{\xi_{T}}{\xi_{t}} \sum_{i=1}^{n}\left(\frac{\lambda}{\beta_{i}} \xi_{T}\right)^{-\frac{1}{\gamma_{i}}} \mid \mathcal{F}_{t}\right] \\
& =G_{t}+\sum_{i=1}^{n} \mathbb{E}\left[\left(\frac{\xi_{T}}{\xi_{t}}\right)^{1-\frac{1}{\gamma_{i}}}\left(\frac{\lambda}{\beta_{i}} \xi_{t}\right)^{-\frac{1}{\gamma_{i}}} \mid \mathcal{F}_{t}\right] \\
& =G_{t}+\sum_{i=1}^{n}\left(\frac{\lambda}{\beta_{i}} \xi_{t}\right)^{-\frac{1}{\gamma_{i}}} k_{i}(t),
\end{aligned}
$$

where $k_{i}(t):=\mathbb{E}\left[\left(\frac{\xi_{T}}{\xi_{t}}\right)^{1-\frac{1}{\gamma_{i}}}\right]=e^{\left(1-\frac{1}{\gamma_{i}}\right)\left(-r-\frac{1}{2} \chi^{2}\right)(T-t)+\frac{1}{2} \chi^{2}\left(1-\frac{1}{\gamma_{i}}\right)^{2}(T-t)}$. Applying Itô's formula to (14) and comparing it to the wealth dynamics in (6), we obtain the self-financing investment strategy by equating the coefficients of $d W_{t}$ :

$$
\pi_{t}^{*}=\sum_{i=1}^{n} \frac{k_{i}(t)}{X_{t}^{*}}\left(\frac{\lambda}{\beta_{i}} \xi_{t}\right)^{-\frac{1}{\gamma_{i}}} \frac{\chi}{\sigma \gamma_{i}}
$$

where the terms $\frac{\chi}{\sigma \gamma_{i}}$ are the individual Merton portfolios (Merton 1971). For the special case $n=1$, expression (15) simplifies as follows for an individual investor $i$ :

$$
\pi_{t}^{(i, *)}=\frac{\chi}{\sigma \gamma_{i}} \cdot \frac{k_{i}(t)\left(\lambda_{i} \xi_{t}\right)^{-\frac{1}{\gamma_{i}}}}{X_{t}^{(i, *)}}=\frac{\chi}{\sigma \gamma_{i}} \cdot \frac{X_{t}^{(i, *)}-G_{t}^{i}}{X_{t}^{(i, *)}}=: m_{i} \cdot \frac{X_{t}^{(i, *)}-G_{t}^{i}}{X_{t}^{(i, *)}},
$$

where $X_{t}^{(i, *)}$ can be obtained from (14) by setting $n=1$. The strategy in (16) is a CPPI strategy, where the multiplier $m_{i}$ is the Merton portfolio of investor $i$. It is a well-known result that the strategy given in (16) is optimal for investors with modified power utility preferences [see, for example, Basak (2002)]. The idea behind a CPPI strategy is simple: To ensure that the guarantee level $G^{i}$ is met, the fraction of wealth $\frac{G_{t}^{i}}{X_{t}^{(i, *)}}$ is invested in the riskfree asset. The remainder $\frac{X_{t}^{(i, *)}-G_{t}^{i}}{X_{t}^{(i, *)}}$, where $X_{t}^{(i, *)}-G_{t}^{i}$ is the so-called cushion, multiplied by $m_{i}$ is then the fraction of wealth invested in the risky asset. Note, however, that the collective optimal solution obtained in (15) is not a CPPI strategy. In this sense, there is a clear difference between the optimal individual and collective investment strategy. There are going to be losses in the collective expected utility if a CPPI investment strategy is applied by a fund manager. It would then be interesting to analyze the suboptimality induced by using CPPI strategy on the individual investors. We leave this analysis for future research. 


\section{Collective investment under stochastic volatility}

As motivated in the introduction, the assumption of a constant volatility may not reflect a realistic financial market. In this section, we describe the evolution of the stock with a more general stochastic volatility model in the sense of Heston (1993). In this stochastic volatility setting, we solve the collective optimal investment problems, based on which we then study the welfare implications to the individual investors.

\subsection{Financial market}

We assume that the volatility of the risky asset is itself driven by a stochastic process. While the risk-free asset $B$ remains as in (5), the drift and the volatility of the risky asset are now given by $\mu_{t}$ and $\sqrt{V_{t}}$. Further, let $\left\{W_{t}^{(1)}\right\}_{t \in[0, T]}$ and $\left\{W_{t}^{(2)}\right\}_{t \in[0, T]}$ be two independent Brownian motions in a probability space $(\Omega, \mathcal{F}, \mathbb{P})$. The risky asset and its volatility are then assumed to follow the Heston model (Heston 1993):

$$
\begin{aligned}
& d S_{t}=S_{t}\left(\mu_{t} d t+\sqrt{V_{t}} d W_{t}^{(1)}\right), \\
& d V_{t}=\kappa\left(\bar{V}-V_{t}\right) d t+\delta \sqrt{V_{t}}\left(\rho d W_{t}^{(1)}+\sqrt{1-\rho^{2}} d W_{t}^{(2)}\right),
\end{aligned}
$$

where $\rho \in(-1,1)$ is a correlation coefficient, $\bar{V}>0$ is the long-run mean for the variance, $\kappa>0$ is the speed of mean reversion and $\delta>0$ is the volatility of the variance. In particular, the variance process follows a square-root process as used in the interest rate model in Cox et al. (1985). To ensure that the variance is almost surely positive at all times, we assume $2 \kappa \bar{V} \geq \delta^{2}$ and $V_{0}>0$ [see Cox et al. (1985)]. The perfect negative/positive correlation implies that the variance risk is fully hedgeable through trading in the underlying asset. In this case, we return to a complete market setting. The solution to this problem is then more similar to the constant volatility case.

The variance process contains the second source of risk which is not traded in the market and cannot be hedged. Therefore, the underlying financial market is incomplete. In other words, the market price for the second source of risk is not uniquely determined. A typical way to proceed is to choose a market price of risk for both sources of randomness and make the considered market artificially complete. This can be done by adding a derivative written on the risky asset to the financial market. This approach is well-known in the literature, see, for example, Liu and Pan (2003), Branger et al. (2008), Branger et al. (2017), Escobar et al. (2018) and Chen et al. (2018). We start by assuming

$$
\frac{\mu_{t}-r}{\sqrt{V_{t}}}=\eta_{1} \sqrt{V_{t}}
$$

and define the volatility risk premium as $\eta_{2} \sqrt{V_{t}}$, where $\eta_{1}$ and $\eta_{2}$ are constants. We set

$$
\widetilde{W}_{t}^{(i)}=W_{t}^{(i)}+\int_{0}^{t} \eta_{i} \sqrt{V_{s}} d s
$$

for $i=1,2$. By Girsanov's theorem, $\widetilde{W}_{t}^{(1)}$ and $\widetilde{W}_{t}^{(2)}$ are independent Brownian motions under the probability measure $\mathbb{P}^{(\eta)}, \eta:=\left(\eta_{1}, \eta_{2}\right)$, which is defined by

$$
\zeta_{t}:=\left.\frac{d \mathbb{P}^{(\eta)}}{d \mathbb{P}}\right|_{\mathcal{F}_{t}}=\exp \left(-\int_{0}^{t} \eta_{1} \sqrt{V_{s}} d W_{s}^{(1)}-\int_{0}^{t} \eta_{2} \sqrt{V_{s}} d W_{s}^{(2)}-\frac{\eta_{1}^{2}+\eta_{2}^{2}}{2} \int_{0}^{t} V_{s} d s\right)
$$


for any $t \in[0, T]$. It is a density, as shown in Chen et al. (2018). Note that for the equivalent martingale measure $\mathbb{P}^{(\eta)}$, the process

$$
\xi_{t}^{(\eta)}=\left.e^{-r t} \frac{d \mathbb{P}^{(\eta)}}{d \mathbb{P}}\right|_{\mathcal{F}_{t}}=e^{-r t} \zeta_{t}, \quad t \in[0, T],
$$

is the corresponding pricing kernel or stochastic discounting process. The value $\xi_{t}^{(\eta)}$ has a similar interpretation as the state price density $\xi_{t}$ in the constant volatility framework: The lower $\xi_{t}^{(\eta)}$ gets, the better the market performs. In the following sections, we express the optimal wealth and investment strategy in terms of $\xi_{t}^{(\eta)}$ and can thus easily interpret their performance under different market states. In this market, for any derivative $O$ on the risky asset with some maturity $T_{1} \leq T$, the no-arbitrage-price at time $t \leq T_{1}$ is now given by

$$
O_{t}:=\mathbb{E}\left[\frac{\xi_{T_{1}}^{(\eta)}}{\xi_{t}^{(\eta)}} O_{T_{1}} \mid \mathcal{F}_{t}\right] .
$$

Now let $g$ be a smooth function such that $O_{t}=g\left(t, S_{t}, V_{t}\right)$ (which exists as $\left\{S_{t}, V_{t}\right\}_{t \in[0, T]}$ is a Markov process). Since $e^{-r t} O_{t}$ is a martingale under $\mathbb{P}^{(\eta)}$, Itô's formula leads to

$$
d O_{t}=r O_{t} d t+g_{S} S_{t} \sqrt{V_{t}} d \widetilde{W}_{t}^{(1)}+\delta g_{V} \sqrt{V_{t}}\left(\rho d \widetilde{W}_{t}^{(1)}+\sqrt{1-\rho^{2}} d \widetilde{W}_{t}^{(2)}\right)
$$

where $g_{S}$ and $g_{V}$ are the first order partial derivatives of $g$ with respect to the risky asset and the variance process. Now let $\pi_{t}$ denote the fraction of wealth invested in the risky asset $S$ and $\phi_{t}$ denote the fraction of wealth invested in the derivative $O$. The remainder $1-\pi_{t}-\phi_{t}$ is invested in the risk-free asset. Assume that $\left\{\pi_{t}, \phi_{t}\right\}_{t \in[0, T]}$ is self-financing. This yields the following dynamics for the collective wealth process $\left\{X_{t}\right\}_{t \in[0, T]}$ :

$$
\begin{aligned}
d X_{t} & =X_{t}\left(r d t+\left(\pi_{t}+\phi_{t} \frac{g_{S} S_{t}+\delta \rho g_{V}}{O_{t}}\right) \sqrt{V_{t}} d \widetilde{W}_{t}^{(1)}+\phi_{t} \frac{g_{V} \delta \sqrt{1-\rho^{2}}}{O_{t}} \sqrt{V_{t}} d \widetilde{W}_{t}^{(2)}\right) \\
& =X_{t}\left(r d t+\Theta_{t}^{(1)} \sqrt{V_{t}} d \widetilde{W}_{t}^{(1)}+\Theta_{t}^{(2)} \sqrt{V_{t}} d \widetilde{W}_{t}^{(2)}\right) \\
& =X_{t}\left(\left(r+\eta_{1} \Theta_{t}^{(1)} V_{t}+\eta_{2} \Theta_{t}^{(2)} V_{t}\right) d t+\Theta_{t}^{(1)} \sqrt{V_{t}} d W_{t}^{(1)}+\Theta_{t}^{(2)} \sqrt{V_{t}} d W_{t}^{(2)}\right),
\end{aligned}
$$

where $X_{0}=x, \Theta_{t}^{(1)}$ is the hedge demand and $\Theta_{t}^{(2)}$ is the speculative demand, following, for example, Liu and Pan (2003) and Chen et al. (2018). The hedge demand reflects the fund manager's position in the risk which is hedgeable by the risky asset. The speculative demand reflects the fund manager's position in the risk which cannot be hedged by trading in the risky asset. In our optimization problem, the hedge and speculative demand will be determined explicitly.

Before proceeding to the following sections, let us first state Lemma 2 which is of major importance when determining the solutions of our optimization problems. 
Lemma 2 Consider the following notations:

$$
\begin{aligned}
\eta_{+} & :=\rho \eta_{1}+\sqrt{1-\rho^{2}} \eta_{2}, \\
\eta_{-} & :=\sqrt{1-\rho^{2}} \eta_{1}-\rho \eta_{2}, \\
q_{i} & :=1-\frac{1}{\gamma_{i}}, \\
a_{i} & :=q_{i} \eta_{+} \delta^{-1}, \\
b_{i} & :=q_{i}\left(\eta_{+} \kappa \delta^{-1}+\frac{\eta_{+}^{2}}{2}+\left(1-q_{i}\right) \frac{\eta_{-}^{2}}{2}\right),
\end{aligned}
$$

and assume that

$$
\kappa^{2}+q_{i}\left(2 \eta_{+} \kappa \delta+\eta_{+}^{2} \delta^{2}+\frac{1}{\gamma_{i}} \eta_{-}^{2} \delta^{2}\right) \geq 0 \text { for all } i=1, \ldots, n .
$$

Then it holds

$$
k_{i}^{(\eta)}(t):=\mathbb{E}\left[\left(\frac{\xi_{T}^{(\eta)}}{\xi_{t}^{(\eta)}}\right)^{q_{i}} \mid \mathcal{F}_{t}\right]=e^{-r q_{i}(T-t)+\kappa \bar{V} a_{i}(T-t)} \Psi_{i}\left(T-t, a_{i}, b_{i}, V_{t}\right)
$$

for all $i=1, \ldots, n$, where

$$
\begin{aligned}
\Psi_{i}\left(s, a_{i}, b_{i}, V_{t}\right) & =\exp \left(-A_{i}(s)-V_{t}\left(B_{i}(s)-a_{i}\right)\right), \\
A_{i}(s) & =-\frac{2 \kappa \bar{V}}{\delta^{2}} \ln \left(\frac{2 \theta_{i} e^{\left(\theta_{i}+\kappa\right) \frac{s}{2}}}{\delta^{2} a_{i}\left(e^{\theta_{i} s}-1\right)+\theta_{i}\left(e^{\theta_{i} s}+1\right)+\kappa\left(e^{\theta_{i} s}-1\right)}\right), \\
B_{i}(s) & =\frac{a_{i}\left(\theta_{i}+\kappa+e^{\theta_{i} s}\left(\theta_{i}-\kappa\right)\right)+2 b_{i}\left(e^{\theta_{i} s}-1\right)}{\delta^{2} a_{i}\left(e^{\theta_{i} s}-1\right)+\theta_{i}\left(e^{\theta_{i} s}+1\right)+\kappa\left(e^{\theta_{i} s}-1\right)}, \\
\theta_{i} & =\sqrt{\kappa^{2}+2 b_{i} \delta^{2}} .
\end{aligned}
$$

Proof See Appendix A.

\subsection{Collective optimization problem}

The collective optimization problem under stochastic volatility can be expressed as:

$$
\max _{\left(\pi_{t}, \phi_{t}\right)_{t \in[0, T]}} \mathbb{E}\left[U_{B, G}\left(X_{T}\right)\right] \quad \text { subject to }(18) .
$$

Let us first mention that optimization for a single investor in a market with stochastic volatility has been considered extensively in the literature, see, for example, Pham (2002), Fleming and Hernández-Hernández (2003), Chacko and Viceira (2005), Kraft (2005) and Liu (2006) using the dynamic programming principle. For power utility functions, a closed-form solution can be obtained by applying a separation technique together with a verification step. This verification procedure is essential to make sure that the value function is finite [see, for example, Kraft (2005)]. To the best of our knowledge, the optimization problem (21) has not yet been considered in the literature. In our collective framework, such a separation technique seems not possible. Hence, dynamic programming does not allow us to achieve an explicit solution to the value function and the investment strategies. Therefore, below, we solve Problem (21) by relying on a martingale approach which results in an explicit solution. 
To this end, we complete the market with an additional hedging instrument as discussed in the previous section. In particular, our objective is the following static problem

$$
\max _{X_{T}} \mathbb{E}\left[U_{B, G}\left(X_{T}\right)\right] \quad \text { subject to } \mathbb{E}\left[\xi_{T}^{(\eta)} X_{T}\right]=x .
$$

In order to proceed with the collective utility maximization problem, we shall examine two integrability conditions similar to (9) and (10) to ensure that the optimization problem (22) is well-defined:

$$
\begin{aligned}
\mathbb{E}\left[\xi_{T}^{(\eta)} I_{B, G}\left(\lambda \xi_{T}^{(\eta)}\right)\right] & <\infty, \\
\mathbb{E}\left[U_{B, G}\left(I_{B, G}\left(\lambda \xi_{T}^{(\eta)}\right)\right)\right] & <\infty,
\end{aligned}
$$

for all $\lambda>0$. Note that assumption (19) is sufficient for both conditions to be fulfilled: For (23), this is straightforward to see. To show (24), we use (4) to obtain

$$
\begin{aligned}
\mathbb{E}\left[U_{B, G}\left(I_{B, G}\left(\lambda \xi_{T}^{(\eta)}\right)\right)\right] & =\mathbb{E}\left[\sum_{i=1}^{n} \beta_{i} U_{i, G^{i}}\left(I_{i, G^{i}}\left(\frac{\lambda}{\beta_{i}} \xi_{T}^{(\eta)}\right)\right)\right] \\
& =\sum_{i=1}^{n} \frac{\beta_{i}}{1-\gamma_{i}} \mathbb{E}\left[\left(\frac{\lambda}{\beta_{i}} \xi_{T}^{(\eta)}\right)^{-\frac{1-\gamma_{i}}{\gamma_{i}}}\right]
\end{aligned}
$$

which leads to (19) again. Thus, roughly speaking, the verification result needed in the context of HJB now boils down to the integrability assumption (24) in the martingale approach.

The solution of Problem (22) can be obtained from the Lagrangian approach as

$$
X_{T}^{*}=I_{B, G}\left(\lambda \xi_{T}^{(\eta)}\right)=\sum_{i=1}^{n} I_{i, G^{i}}\left(\frac{\lambda}{\beta_{i}} \xi_{T}^{(\eta)}\right)=\sum_{i=1}^{n}\left(G^{i}+\left(\frac{\lambda}{\beta_{i}} \xi_{T}^{(\eta)}\right)^{-\frac{1}{\gamma_{i}}}\right)
$$

where $\lambda$ is determined from the budget constraint.

Remark 2 For $n=1$, Problem (22) is reduced to the individual optimization problem (taking individual $i$ with an initial wealth $x_{i}>G^{i} e^{-r T}$ as an example):

$$
\max _{X_{T}^{i}} \mathbb{E}\left[U_{i, G_{i}}\left(X_{T}^{i}\right)\right] \text { subject to } \mathbb{E}\left[\xi_{T}^{(\eta)} X_{T}^{i}\right]=x_{i} .
$$

The individual optimal solution for individual i is given by

$$
X_{T}^{(i, *)}=I_{i, G^{i}}\left(\lambda_{i} \xi_{T}^{(\eta)}\right),
$$

where $\lambda_{i}$ can be determined explicitly from the budget constraint and is given by

$$
\lambda_{i}=\left(\frac{x_{i}-G_{0}^{i}}{\mathbb{E}\left[\left(\xi_{T}^{(\eta)}\right)^{1-\frac{1}{\gamma_{i}}}\right]}\right)^{-\gamma_{i}} .
$$

Using Lemma 2, we can now determine the optimal strategy of Problem (22) explicitly. 
Proposition 1 Consider the optimization problem (22). Using the notations in Lemma 2, the optimal wealth at time $t \in[0, T)$ is given by

$$
X_{t}^{*}=X_{t}^{*}\left(\xi_{t}^{(\eta)}, V_{t}\right)=\sum_{i=1}^{n} G_{t}^{i}+\left(\frac{\lambda}{\beta_{i}} \xi_{t}^{(\eta)}\right)^{-\frac{1}{\gamma_{i}}} k_{i}^{(\eta)}(t) .
$$

The optimal hedge and speculative demand are then given by

$$
\begin{aligned}
& \Theta_{t}^{(1, *)}=\frac{1}{X_{t}^{*}} \sum_{i=1}^{n}\left(\frac{\eta_{1}}{\gamma_{i}}-\delta \rho H_{i}(T-t)\right)\left(\frac{\lambda}{\beta_{i}} \xi_{t}^{(\eta)}\right)^{-\frac{1}{\gamma_{i}}} k_{i}^{(\eta)}(t), \\
& \Theta_{t}^{(2, *)}=\frac{1}{X_{t}^{*}} \sum_{i=1}^{n}\left(\frac{\eta_{2}}{\gamma_{i}}-\delta \sqrt{1-\rho^{2}} H_{i}(T-t)\right)\left(\frac{\lambda}{\beta_{i}} \xi_{t}^{(\eta)}\right)^{-\frac{1}{\gamma_{i}}} k_{i}^{(\eta)}(t),
\end{aligned}
$$

where $H_{i}(s):=B_{i}(s)-a_{i}$. In particular, the optimal fraction of wealth invested in the derivative and the risky asset at time $t \in[0, T)$ are then given by

$$
\begin{aligned}
\phi_{t}^{*} & =\frac{\sum_{i=1}^{n}\left(\frac{\eta_{2}}{\gamma_{i}}-\delta \sqrt{1-\rho^{2}} H_{i}(T-t)\right)\left(\frac{\lambda}{\beta_{i}} \xi_{t}^{(\eta)}\right)^{-\frac{1}{\gamma_{i}}} k_{i}^{(\eta)}(t)}{X_{t}^{*} \frac{g_{V} \delta \sqrt{1-\rho^{2}}}{O_{t}}}, \\
\pi_{t}^{*} & =\frac{1}{X_{t}^{*}} \sum_{i=1}^{n}\left(\frac{\eta_{1}}{\gamma_{i}}-\delta \rho H_{i}(T-t)\right)\left(\frac{\lambda}{\beta_{i}} \xi_{t}^{(\eta)}\right)^{-\frac{1}{\gamma_{i}}} k_{i}^{(\eta)}(t)-\phi_{t}^{*} \frac{g_{S} S_{t}+\delta \rho g_{V}}{O_{t}} .
\end{aligned}
$$

Proof Using Lemma 2, we obtain

$$
\begin{aligned}
X_{t}^{*} & =\mathbb{E}\left[\frac{\xi_{T}^{(\eta)}}{\xi_{t}^{(\eta)}} I_{B, G}\left(\lambda \xi_{T}^{(\eta)}\right) \mid \mathcal{F}_{t}\right]=\sum_{i=1}^{n} \mathbb{E}\left[\frac{\xi_{T}^{(\eta)}}{\xi_{t}^{(\eta)}}\left(G^{i}+\left(\frac{\lambda}{\beta_{i}} \xi_{T}^{(\eta)}\right)^{-\frac{1}{\gamma_{i}}}\right) \mid \mathcal{F}_{t}\right] \\
& =\sum_{i=1}^{n} G_{t}^{i}+\left(\frac{\lambda}{\beta_{i}} \xi_{t}^{(\eta)}\right)^{-\frac{1}{\gamma_{i}}} k_{i}^{(\eta)}(t) .
\end{aligned}
$$

Next, we compute the optimal fractions of wealth invested in the risky asset $\pi_{t}^{*}$ and the derivative $\phi_{t}^{*}$. Observe that

$$
\begin{aligned}
\frac{\partial X_{t}^{*}}{\partial \xi_{t}^{(\eta)}} & =-\sum_{i=1}^{n} \frac{1}{\gamma_{i}}\left(\frac{\lambda}{\beta_{i}}\right)^{-\frac{1}{\gamma_{i}}}\left(\xi_{t}^{(\eta)}\right)^{-\frac{1}{\gamma_{i}}-1} k_{i}^{(\eta)}(t), \\
\frac{\partial X_{t}^{*}}{\partial V_{t}} & =-\sum_{i=1}^{n}\left(\frac{\lambda}{\beta_{i}} \xi_{t}^{(\eta)}\right)^{-\frac{1}{\gamma_{i}}} k_{i}^{(\eta)}(t) H_{i}(T-t) .
\end{aligned}
$$

We use Itô's formula to represent the dynamics of the wealth process as

$$
d X_{t}^{*}=(\mathrm{drift}) d t+\frac{\partial X_{t}^{*}}{\partial \xi_{t}^{(\eta)}} d \xi_{t}^{(\eta)}+\frac{\partial X_{t}^{*}}{\partial V_{t}} d V_{t} .
$$

Recall that

$$
\begin{aligned}
d \xi_{t}^{(\eta)} & =-\xi_{t}^{(\eta)}\left(r d t+\eta_{1} \sqrt{V_{t}} d W_{t}^{(1)}+\eta_{2} \sqrt{V_{t}} d W_{t}^{(2)}\right), \\
d V_{t} & =\kappa\left(\bar{V}-V_{t}\right) d t+\delta \sqrt{V_{t}}\left(\rho d W_{t}^{(1)}+\sqrt{1-\rho^{2}} d W_{t}^{(2)}\right) .
\end{aligned}
$$


This leads to

$$
\begin{aligned}
\frac{\partial X_{t}^{*}}{\partial \xi_{t}^{(\eta)}} d \xi_{t}^{(\eta)}= & \sum_{i=1}^{n} \frac{1}{\gamma_{i}}\left(\frac{\lambda}{\beta_{i}} \xi_{t}^{(\eta)}\right)^{-\frac{1}{\gamma_{i}}} k_{i}^{(\eta)}(t)\left(r d t+\eta_{1} \sqrt{V_{t}} d W_{t}^{(1)}+\eta_{2} \sqrt{V_{t}} d W_{t}^{(2)}\right) \\
\frac{\partial X_{t}^{*}}{\partial V_{t}} d V_{t}= & -\sum_{i=1}^{n}\left(\frac{\lambda}{\beta_{i}} \xi_{t}^{(\eta)}\right)^{-\frac{1}{\gamma_{i}}} k_{i}^{(\eta)}(t) H_{i}(T-t) \\
& \left(\kappa\left(\bar{V}-V_{t}\right) d t+\delta \sqrt{V_{t}}\left(\rho d W_{t}^{(1)}+\sqrt{1-\rho^{2}} d W_{t}^{(2)}\right)\right) .
\end{aligned}
$$

From this, together with (18), we obtain

$$
\begin{aligned}
& \Theta_{t}^{(1, *)} X_{t}^{*} \sqrt{V_{t}} d W_{t}^{(1)}=\sum_{i=1}^{n}\left(\frac{\eta_{1}}{\gamma_{i}}-\delta \rho H_{i}(T-t)\right)\left(\frac{\lambda}{\beta_{i}} \xi_{t}^{(\eta)}\right)^{-\frac{1}{\gamma_{i}}} k_{i}^{(\eta)}(t) \sqrt{V_{t}} d W_{t}^{(1)}, \\
& \Theta_{t}^{(2, *)} X_{t}^{*} \sqrt{V_{t}} d W_{t}^{(2)}=\sum_{i=1}^{n}\left(\frac{\eta_{2}}{\gamma_{i}}-\delta \sqrt{1-\rho^{2}} H_{i}(T-t)\right)\left(\frac{\lambda}{\beta_{i}} \xi_{t}^{(\eta)}\right)^{-\frac{1}{\gamma_{i}}} k_{i}^{(\eta)}(t) \sqrt{V_{t}} d W_{t}^{(2)} .
\end{aligned}
$$

From the definitions of $\Theta_{t}^{(1)}$ and $\Theta_{t}^{(2)}$ as given in (18), it is straightforward to derive formulas for $\pi_{t}^{*}$ and $\phi_{t}^{*}$ :

$$
\begin{aligned}
\Theta_{t}^{(1)} X_{t}^{*} & =\left(\pi_{t}+\phi_{t} \frac{g_{S} S_{t}+\delta \rho g_{V}}{O_{t}}\right) X_{t}^{*}=\sum_{i=1}^{n}\left(\frac{\eta_{1}}{\gamma_{i}}-\delta \rho H_{i}(T-t)\right)\left(\frac{\lambda}{\beta_{i}} \xi_{t}^{(\eta)}\right)^{-\frac{1}{\gamma_{i}}} k_{i}^{(\eta)}(t) \\
& \Leftrightarrow \pi_{t}=\frac{1}{X_{t}^{*}} \sum_{i=1}^{n}\left(\frac{\eta_{1}}{\gamma_{i}}-\delta \rho H_{i}(T-t)\right)\left(\frac{\lambda}{\beta_{i}} \xi_{t}^{(\eta)}\right)^{-\frac{1}{\gamma_{i}}} k_{i}^{(\eta)}(t)-\phi_{t} \frac{g_{S} S_{t}+\delta \rho g_{V}}{O_{t}} \\
\Theta_{t}^{(2)} X_{t}^{*} & =\phi_{t} \frac{g_{V} \delta \sqrt{1-\rho^{2}}}{O_{t}} X_{t}^{*}=\sum_{i=1}^{n}\left(\frac{\eta_{2}}{\gamma_{i}}-\delta \sqrt{1-\rho^{2}} H_{i}(T-t)\right)\left(\frac{\lambda}{\beta_{i}} \xi_{t}^{(\eta)}\right)^{-\frac{1}{\gamma_{i}}} k_{i}^{(\eta)}(t) \\
& \Leftrightarrow \phi_{t}=\frac{\sum_{i=1}^{n}\left(\frac{\eta_{2}}{\gamma_{i}}-\delta \sqrt{1-\rho^{2}} H_{i}(T-t)\right)\left(\frac{\lambda}{\beta_{i}} \xi_{t}^{(\eta)}\right)^{-\frac{1}{\gamma_{i}}} k_{i}^{(\eta)}(t)}{\frac{g_{V} \delta \sqrt{1-\rho^{2}}}{O_{t}} X_{t}^{*}}
\end{aligned}
$$

Remark 3 As pointed out in Chen et al. (2018) (Appendix F), individual $i$ 's optimal hedge and speculative demand in the case without guarantees are given by $\frac{\eta_{1}}{\gamma_{i}}-\delta \rho H_{i}(T-t)$ and $\frac{\eta_{2}}{\gamma_{i}}-\delta \sqrt{1-\rho^{2}} H_{i}(T-t)$, respectively. Hence, the collective optimal hedge and speculative demand are given as the sum of the optimal individual demands without guarantees, weighted by $\left(\frac{\lambda}{\beta_{i}} \xi_{t}^{(\eta)}\right)^{-\frac{1}{\gamma_{i}}} k_{i}^{(\eta)}(t) / X_{t}^{*}$, which is individual $i$ 's surplus [see (30) for $n=1$ ] divided by the collective optimal wealth.

Let us consider a numerical example. The base case parameter choice is summarized in Table 1. Concerning the choice of the volatility parameters and the correlation, we follow Liu and Pan (2003) whose choice of parameters is "in the generally agreed region" of the empirical studies by Andersen et al. (2002), Pan (2002) and Eraker et al. (2003). Concerning the risk premiums, we also follow Liu and Pan (2003). In particular, we assume that volatility risk is negatively priced, as supported by the findings of Benzoni (1998), Chernov and Ghysels 
Table 1 Parameters of the standard example

\begin{tabular}{lll}
\hline $\begin{array}{l}\text { Volatility parameters } \\
V_{0}=\bar{V}=0.13^{2}, \kappa=5, \delta=0.25\end{array}$ & $\begin{array}{l}\text { Risk premiums } \\
\eta_{1}=4, \eta_{2}=-6\end{array}$ & $\begin{array}{l}\text { Correlation } \\
\rho=-0.4\end{array}$ \\
\hline Risk-free rate, maturity & Pool size & Weights $\beta_{i}$ \\
$r=0.02, T=1$ & $n=30$ & $\beta_{i}=\frac{\left(\lambda_{i}\right)^{-1}}{\sum_{i=1}^{n}\left(\lambda_{i}\right)^{-1}}, i=1, \ldots, n$ \\
\hline Degrees of risk aversion & Initial wealth & Guarantee \\
$\gamma_{i}=\frac{1}{2}+\frac{9.5(i-1)}{n-1}, i=1, \ldots, n$ & $x_{i}=1, i=1, \ldots, n$ & $G_{i}=0.5, i=1, \ldots, n$ \\
\hline
\end{tabular}

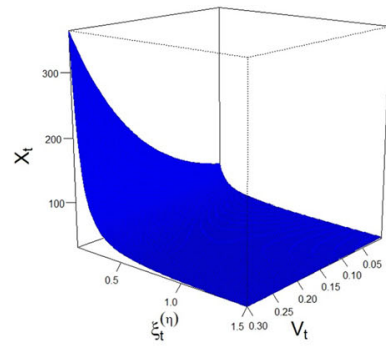

(a) Optimal wealth

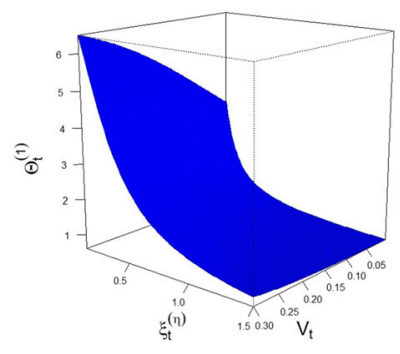

(b) Hedge demand

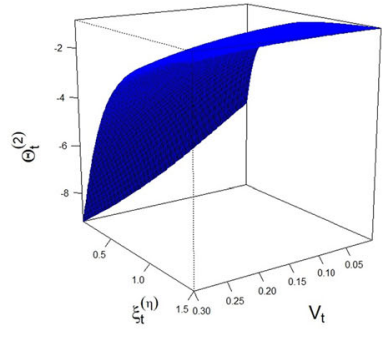

(c) Speculative demand

Fig. 1 The optimal wealth $X_{t}^{*}$, the hedge demand $\Theta_{t}^{(1, *)}$ and the speculative demand $\Theta_{t}^{(2, *)}$ as functions of the pricing kernel $\xi_{t}^{(\eta)}$ and the variance $V_{t}$ at $t=T / 2$ for the base case parameter setup summarized in Table 1

(2000), Pan (2002) and Bakshi and Kapadia (2003). Although we rely on the same financial market parameters as Liu and Pan (2003), the goals of ours differ substantially from theirs, which makes a direct comparison of these two papers rather difficult. While Liu and Pan (2003) focus on the portfolio improvement from participating in the derivatives market using the HJB approach, we are interested in risk sharing and welfare analysis of the collective in a stochastic volatility market using a martingale approach. We consider a collective of investors with heterogeneous risk preferences, rather than a single investor as in Liu and Pan (2003). In addition, we consider HARA-type utility functions taking into account a demand for guarantee in the investment decision, whereas Liu and Pan (2003) apply CRRA-utility preferences. Due to the last two points, some parts of our model setup are more general than the one in Liu and Pan (2003), while some parts are less. For instance, Liu and Pan (2003) also consider jump risk in their model, while we focus exclusively on the volatility risk. Note that the negative price of volatility risk leads the investor to seek a short position in volatility risk. In other words, under negatively priced volatility risk, an investor seeks a short position in derivatives with positive exposure to the volatility risk. The choice of the weights $\beta_{i}$ is motivated by Sect. 5, where we show that the collective terminal wealth (25) rewrites to the sum of individual terminal wealths (27) under these weights.

In Fig. 1 the optimal wealth at time $t=1 / 2$, the hedge demand and the speculative demand are plotted as functions of the pricing kernel $\xi_{t}^{(\eta)}$ and the instantaneous variance $V_{t}$ at $t=1 / 2$.

We see that the optimal wealth and the hedge demand are increasing in the variance $V_{t}$ and that the speculative demand is decreasing in $V_{t}$. The reason for the increase of the wealth and the hedge demand in this example is assumption (17) along with the positive 
choice of $\eta_{1}$ which imply that an increase in the volatility at time $t$ yields a higher rate of return per unit of volatility. The decrease of the speculative demand can be explained by the negatively priced volatility risk $\left(\eta_{2}<0\right)$. Regarding the pricing kernel, we observe that well-performing markets (a low value of $\xi_{t}^{(\eta)}$ ) lead to a higher wealth and hedge demand and a lower speculative demand. Further, we see that the hedge demand is positive (long position) while the speculative demand is negative (short position) in all scenarios. The reason for this "reverse" behavior of the hedge and speculative demand in our parameter setup is the negative volatility risk premium which results from choosing $\eta_{2}$ smaller than zero.

\section{Achieving individual optimal solutions}

In this section, we address the question how the optimal terminal wealth (25) can be shared among the individuals in the collective. As it is the fund manager's primal goal to meet individual guarantees $G^{i}$, we assume that the fund manager starts by distributing to each individual her guarantee $G^{i}$. Further, let $\left(\alpha_{i}\right)_{i=1, \ldots, n}$ be any (possibly state-dependent) sharing rule satisfying $\alpha_{i} \geq 0$ for all $i=1, \ldots, n$ and $\sum_{i=1}^{n} \alpha_{i}=1$. This sharing rule is applied to the wealth exceeding the collective guarantee and determines the fraction of terminal surplus each individual receives. That is, for any collective terminal wealth $X_{T}>G$, investor $i$ receives $X_{T}^{i}=G^{i}+\alpha_{i}\left(X_{T}-G\right)$. Based on the optimal collective terminal wealth (25), a natural candidate for the terminal wealth which investor $i$ obtains is given by

$$
X_{T}^{i}=I_{i, G^{i}}\left(\frac{\lambda}{\beta_{i}} \xi_{T}^{(\eta)}\right)=G^{i}+\left(\frac{\lambda}{\beta_{i}} \xi_{T}^{(\eta)}\right)^{-\frac{1}{\gamma_{i}}},
$$

that is,

$$
\alpha_{i}\left(\xi_{T}^{(\eta)}\right)=\frac{\left(\frac{\lambda}{\beta_{i}} \xi_{T}^{(\eta)}\right)^{-\frac{1}{\gamma_{i}}}}{X_{T}^{*}}
$$

Naturally, the question arises whether a fair way of sharing the surplus can be achieved by a specific choice of the weights $\beta_{i}$, as the sharing rule in (32) is not necessarily fair. Without fairness, there might be some individuals in the collective who profit from the collective investment and some who suffer losses (compared to their individual investment). Let us now assume that the financial fairness condition as considered in Bühlmann and Jewell (1979) or, more recently, also in Schumacher (2018) is fulfilled. To be precise, we assume that the initial market value of the terminal payoff received by each investor $i$ equals the initial contribution of this investor, that is,

$$
x_{i}=\mathbb{E}\left[\xi_{T}^{(\eta)} I_{i, G^{i}}\left(\frac{\lambda}{\beta_{i}} \xi_{T}^{(\eta)}\right)\right] .
$$

In our setting, it is then possible to return to each investor her individual optimum as obtained from Problem (22) for $n=1$.

Proposition 2 We assume that each investor in the collective receives the terminal wealth given in (31). If we further impose the financial fairness condition (33), each investor in the collective obtains her individual optimum as given in (27). 
Proof Let us introduce the notation $k_{i}^{(\eta)}=k_{i}^{(\eta)}(0)$ as defined in equation (20). The financial fairness condition delivers

$$
x_{i}=\mathbb{E}\left[\xi_{T}^{(\eta)} I_{i, G^{i}}\left(\frac{\lambda}{\beta_{i}} \xi_{T}^{(\eta)}\right)\right]=G_{0}^{i}+\left(\frac{\lambda}{\beta_{i}}\right)^{-\frac{1}{\gamma_{i}}} k_{i}^{(\eta)} \Leftrightarrow \frac{\beta_{i}}{\lambda}=\left(\frac{x_{i}-G_{0}^{i}}{k_{i}^{(\eta)}}\right)^{\gamma_{i}} .
$$

Using the fact that the $\beta_{i}$ add up to 1 , we obtain

$$
\lambda=\frac{1}{\sum_{i=1}^{n}\left(\frac{x_{i}-G_{0}^{i}}{k_{i}^{(\eta)}}\right)^{\gamma_{i}}}, \quad \beta_{i}=\frac{\left(\frac{x_{i}-G_{0}^{i}}{k_{i}^{(\eta)}}\right)^{\gamma_{i}}}{\sum_{j=1}^{n}\left(\frac{x_{j}-G_{0}^{j}}{k_{j}^{(\eta)}}\right)^{\gamma_{j}}} .
$$

For the case $n=1$, the budget constraint of Problem (22) can be written as

$$
x_{i}=\mathbb{E}\left[\xi_{T}^{(\eta)} I_{i, G^{i}}\left(\lambda_{i} \xi_{T}^{(\eta)}\right)\right]=G_{0}^{i}+\lambda_{i}^{-\frac{1}{\gamma_{i}}} k_{i}^{(\eta)} .
$$

Plugging this expression into the two expressions given in (34), we obtain

$$
\lambda=\frac{1}{\sum_{i=1}^{n}\left(\lambda_{i}\right)^{-1}}, \quad \beta_{i}=\frac{\left(\lambda_{i}\right)^{-1}}{\sum_{i=1}^{n}\left(\lambda_{i}\right)^{-1}} .
$$

Consequently, each investor's terminal payoff in (31) simplifies to the following:

$$
X_{T}^{i}=G^{i}+\left(\frac{\lambda}{\beta_{i}} \xi_{T}^{(\eta)}\right)^{-\frac{1}{\gamma_{i}}}=G^{i}+\left(\lambda_{i} \xi_{T}^{(\eta)}\right)^{-\frac{1}{\gamma_{i}}}=I_{i, G^{i}}\left(\lambda_{i} \xi_{T}^{\eta}\right)
$$

where $I_{i, G^{i}}\left(\lambda_{i} \xi_{T}^{(\eta)}\right)$ is the individual optimum given in (27).

Proposition 2 states that it is possible to achieve the individual optimal terminal wealth for all the individuals in the collective. The main assumptions for this result are the financial fairness and the use of a state-dependent sharing rule. This result is also valid under constant volatility and can be proven in the exact same way by simply replacing $\xi_{T}^{(\eta)}$ with $\xi_{T}$. In fact, this result has already been proven in Branger et al. (2018b) in a Black-Scholes market for CRRA utility functions (i.e. with all the individual guarantees being equal to zero).

Under the financial fairness condition, the sharing rule (32) can be simplified to the following:

$$
\alpha_{i}\left(\xi_{T}^{(\eta)}\right)=\frac{\left(\lambda_{i} \xi_{T}^{(\eta)}\right)^{-\frac{1}{\gamma_{i}}}}{\sum_{j=1}^{n}\left(\lambda_{j} \xi_{T}^{(\eta)}\right)^{-\frac{1}{\gamma_{j}}}}
$$

for all $i=1, \ldots, n$. A disadvantage of this sharing rule is, however, that it depends on the market state at maturity and is, thus, not easy to communicate. Therefore, in the following section, we consider two examples of more prevailing sharing rules which are easier to communicate.

\section{Linear sharing rules and welfare analysis}

In practice, sharing rules that are easier to communicate, like linear (or affine) sharing rules, are applied. We aim at finding out how these sharing rules affect individuals' benefits in a 
stochastic volatility setting and compare the results to a constant volatility setting. Following the existing literature, we consider two further sharing rules in addition to the sharing rule defined in (36). The sharing rules considered here are inspired by previous works concerning a collective of individuals facing a joint decision under uncertainty. For example, Wilson (1968) and Huang and Litzenberger (1985) analyze the Pareto optimality of sharing rules. Weinbaum (2009) considers two individuals with different utility functions who are tied together by a social planner who uses a weighted sum of the individual utility functions and then characterizes the optimal sharing rule implicitly. Jensen and Nielsen (2016) consider a similar social planner whose sharing rule is initially fixed to be linear, though. Branger et al. (2018a) consider a rather similar setting as Jensen and Nielsen (2016) but generalize the analysis to $n$ investors instead of two.

- Linear sharing rule (without financial fairness): One of the most frequently used sharing rules in practice is the linear (or affine) sharing rule $\left(\widetilde{\alpha}_{i}\right)_{i=1, \ldots, n}$ defined by

$$
\tilde{\alpha_{i}}=\frac{x_{i}}{x}, \quad i=1, \ldots, n
$$

The shares that the individuals obtain from the total surpluses correspond to the shares of their initial investment in the fund. This simple sharing rule is known at time 0 and is thus easier to communicate than the state-dependent sharing rule (36). In addition, it can be shown that this linear sharing rule does not necessarily fulfill the financial fairness condition. It has been documented that this linear sharing rule is suboptimal, see, for example, Jensen and Nielsen (2016) and Branger et al. (2018a). In our numerical analysis, we will quantify the possible utility loss for the individuals.

- Financially fair linear sharing rule: A slight modification of (37) delivers the fair sharing rule $\left(\widehat{\alpha}_{i}\right)_{i=1, \ldots, n}$ which is defined as

$$
\widehat{\alpha}_{i}=\frac{x_{i}-G_{0}^{i}}{x-G_{0}}, \quad i=1, \ldots, n .
$$

It is straightforward to check that this sharing rule results in a financially fair payoff to each individual.

Note that none of the two linear sharing rules manages to deliver the individually optimal solutions to all the individuals in the collective. In the following, we compare the well-being of the investors in the financial market under the sharing rules introduced above. To measure the well-being of the investors, we rely on the certainty equivalent return introduced in Sect. 6.1. For our analysis, we assume that the weights $\beta_{i}$ are given as in (35).

\subsection{Certainty equivalent}

For any investor $i$ and a given terminal payoff $X_{T}^{i}$, the certainty equivalent wealth is denoted by $\mathrm{CE}_{i}=\mathrm{CE}_{i}\left(X_{T}^{i}\right)$. It is defined as the deterministic wealth level which yields the same expected utility as some terminal wealth $X_{T}^{i}$ :

$$
U_{i, G^{i}}\left(\mathrm{CE}_{i}\right)=\mathbb{E}\left[U_{i, G^{i}}\left(X_{T}^{i}\right)\right] .
$$

This results in

$$
\mathrm{CE}_{i}=\mathbb{E}\left[\left(\alpha_{i}\left(X_{T}^{*}-G\right)\right)^{1-\gamma_{i}}\right]^{\frac{1}{1-\gamma_{i}}}+G^{i}
$$


for some sharing rule $\left(\alpha_{i}\right)_{i=1, \ldots, n}$. Under the state-dependent sharing rule (36), we can compute the certainty equivalent of investor $i$ in the Heston model as

$$
\begin{aligned}
\mathrm{CE}_{i}^{*} & =G^{i}+\mathbb{E}\left[\left(\lambda_{i} \xi_{T}^{(\eta)}\right)^{-\frac{1-\gamma_{i}}{\gamma_{i}}}\right]^{\frac{1}{1-\gamma_{i}}} \\
& =G^{i}+\lambda_{i}^{-\frac{1}{\gamma_{i}}} \mathbb{E}\left[\left(\xi_{T}^{(\eta)}\right)^{1-\frac{1}{\gamma_{i}}}\right]^{\frac{1}{1-\gamma_{i}}} \\
& =G^{i}+\lambda_{i}^{-\frac{1}{\gamma_{i}}}\left(k_{i}^{(\eta)}\right)^{\frac{1}{1-\gamma_{i}}} .
\end{aligned}
$$

Under the linear sharing rule (37), for example, we can compute the certainty equivalent of investor $i$ in the Heston model as

$$
\begin{aligned}
\widetilde{\mathrm{CE}}_{i} & =\mathbb{E}\left[\left(\widetilde{\alpha}_{i}\left(X_{T}^{*}-G\right)\right)^{1-\gamma_{i}}\right]^{\frac{1}{1-\gamma_{i}}}+G^{i} \\
& =\widetilde{\alpha}_{i} \mathbb{E}\left[\left(X_{T}^{*}-G\right)^{1-\gamma_{i}}\right]^{\frac{1}{1-\gamma_{i}}}+G^{i} \\
& =\widetilde{\alpha}_{i} \mathbb{E}\left[\left(\sum_{j=1}^{n}\left(\lambda_{j} \xi_{T}^{(\eta)}\right)^{-\frac{1}{\gamma_{j}}}\right)^{1-\gamma_{i}}\right]^{\frac{1}{1-\gamma_{i}}}+G^{i},
\end{aligned}
$$

and an analogous calculation can be carried out for the linear sharing rule (38). Inspired by, for example, Zieling et al. (2014) and Branger et al. (2018a), we now consider the certainty equivalent return. It is defined as the deterministic rate of return $y_{i}=y_{i}\left(X_{T}^{i}\right)$ which delivers the same utility as some state-dependent terminal wealth, that is,

$$
U_{i, G^{i}}\left(x_{i} e^{y_{i} T}\right)=\mathbb{E}\left[U_{i, G^{i}}\left(X_{T}^{i}\right)\right] \quad \Leftrightarrow \quad y_{i}=\frac{1}{T} \ln \left(\frac{\mathrm{CE}_{i}\left(X_{T}^{i}\right)}{x_{i}}\right) .
$$

The certainty equivalent return is easier to interpret than the certainty equivalent wealth, particularly when individuals own different wealth levels.

\subsection{Numerical analysis}

In Fig. 2, we compare the certainty equivalent returns defined in (41). Panel (a) demonstrates the certainty equivalent returns for the base case, where the parameters are listed in Table 1. In particular, we have used in the base case that the initial wealth levels and the required guarantees for all the individuals are identical, which implies that both linear sharing rules are identical. In addition to the base case, we show in Panel (b) the case of a guarantee which increases in $\gamma_{i}$. The guarantees for this case are chosen as

$$
G^{i}=p_{i} x_{i} e^{g_{i} T}, \quad p_{i}=\frac{i-1}{n-1}, \quad g_{i}=-0.015+0.03 \frac{i-1}{n-1}, \quad i=1, \ldots, n .
$$

This illustrates a more realistic choice for the minimum guarantees: the more risk-averse an individual is, the higher is the minimum guarantee chosen.

Note that in the determination of the optimal collective investment strategies, only the total guarantee level plays a role, while the individuals' certainty equivalent returns do additionally depend on the individual guarantees. In Fig. 2, we observe the following: 


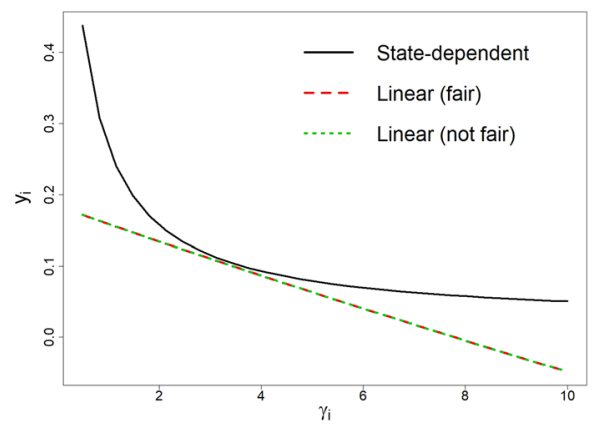

(a) Base case

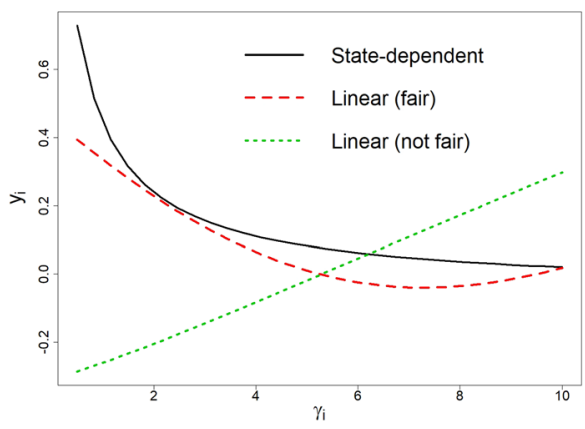

(b) Base case with increasing guarantee

Fig. 2 Certainty equivalent returns (41) of the investors in the collective. In Panel (a), the base case is used, while in Panel (b), we show the case given in (42)

- In Panel (a), we observe that imposing the linear sharing rule leads to losses for all the individuals, which can be considered as the (negative) deviation of the certainty equivalent returns from the state-dependent case (or individuals' optimal certainty equivalent returns). High losses arise for the least and the most risk-averse individuals. The benefits of some investors are hardly influenced by the linear sharing rule. The main reason for this result is probably that the optimal collective investment strategy is closest to the individual optimal investment strategies with $\gamma_{i}$ around 3.5. For those who are least or most risk-averse, the optimal collective investment strategy differs significantly from their individual optimal investment strategies.

- In Panel (b), we make the following observations:

- Fair linear sharing rule: Similarly as in Panel (a), compared to the (fair) statedependent sharing rule, the application of a fair linear sharing rule causes losses to all the investors. Different from Panel (a), individuals having medium and small risk aversions suffer most. The reason that highly risk-averse investors' losses are reduced compared to Panel (a) is the additional effect caused by their required high minimum guarantees.

- Unfair linear sharing rule: As the fund manager will first meet all the individual guarantees and then split the surpluses, individuals who require low guarantees implicitly finance the guarantees of individuals who demand high guarantees. For the unfair linear sharing rule, this argument seems to dominate. As a consequence, individuals demanding low guarantees suffer drastic losses, whereas investors demanding high guarantees are better off compared to their individual optimal solution. The certainty equivalents can even become negative for individuals with low risk aversions and low guarantees.

Due to the drastic losses occurring under unfair linear sharing rules (compared to moderate losses under a fair sharing rule), fairness shall certainly be taken into consideration if a linear sharing rule is applied in practice.

In Fig. 3 we compare the certainty equivalents of the investors in the collective under constant volatility. We use the parameters from Table 1 . The parameters of the Black-Scholes market are specified as $\mu=0.0876$ and $\sigma=0.13$. Note that we obtain $\frac{\mu-r}{\sigma}=\eta_{1} \sqrt{\bar{V}}$ under these parameters. We consider the Black-Scholes analogue of the sharing rule defined in (36) (that is, we replace $\xi_{T}^{(\eta)}$ by $\xi_{T}$ ) and the linear sharing rules (37) and (38). 


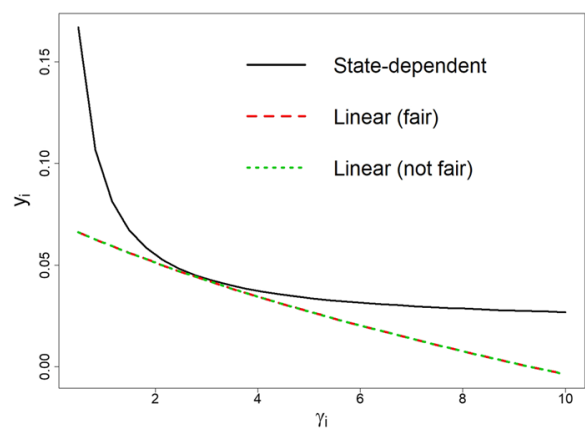

(a) Base case

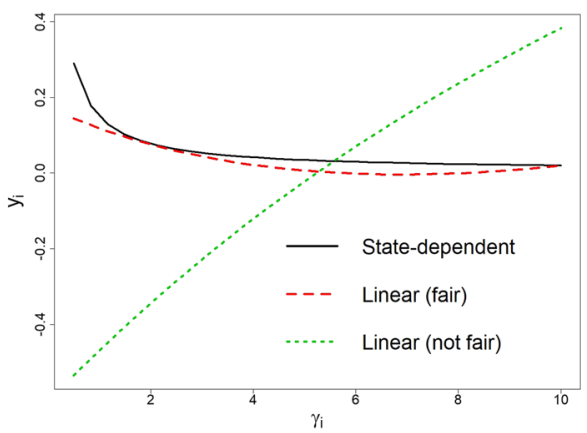

(b) Base case with increasing guarantee

Fig. 3 Certainty equivalent returns (41) of the investors in the collective for constant volatility case (where $\xi_{T}^{(\eta)}$ is replaced by $\xi_{T}$ ). In Panel (a), the base case is used, while in Panel (b), we show the case (42)

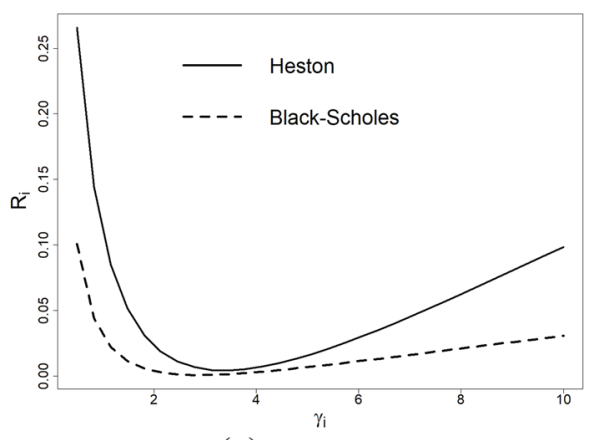

(a) Base case

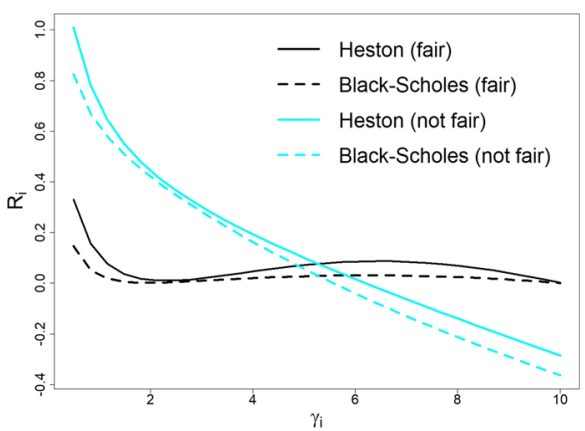

(b) Base case with increasing guarantee

Fig. 4 Relative losses $R_{i}$ as defined in (43) of the investors in the collective. In Panel (a), the base case is used, while in Panel (b), we show the case (42)

We observe from Fig. 3 that the certainty equivalent returns under constant volatility exhibit similar patterns as in the stochastic volatility case (cf. Fig. 2). However, the certainty equivalent returns under constant volatility seem to be overall lower than in the stochastic volatility case. The "unfairness" caused by the (unfair) linear sharing rule (37) seems to weaken slightly.

In conclusion, by the use of the unfair linear sharing rule, individuals requiring high guarantees benefit largely from the collective, while those who require low guarantees suffer substantially from the collective. The fair linear sharing rule, on the other hand, causes only moderate losses to all the individuals in the collective. Thus, from a fund manager's perspective, to serve each individual in the collective fairly, the use of a financially fair sharing rule shall be preferred.

Given the widespread use of linear sharing rules, it is interesting to find out whether the sub-optimality of linear sharing rules will be amplified in the more realistic stochastic volatility setting. For this purpose, we consider the quantity

$$
R_{i}:=y_{i}^{*}-y_{i}^{\ell}
$$

for all individuals $i=1, \ldots, n$, where $y_{i}^{\ell}$ is the certainty equivalent return under a linear sharing rule. They are provided in Fig. 4. 
In both panels, we observe that the curves resulting from the Heston model lie above those from the Black-Scholes model. In other words, the imposition of a suboptimal linear sharing rule leads to larger losses (and, in Panel (b), smaller gains) for all the individuals in the collective under the Heston model compared to the Black-Scholes model. Let us, for example, consider the base case: In the current parameter choice, an individual with parameter $\gamma_{i} \approx 6$ obtains a certainty equivalent return that is $3 \%$ lower than the optimal one in the Heston model and 1\% lower than the one in the Black-Scholes model. Among the individuals in the collective, the difference between the two models is the largest for those with a very low risk aversion. This behavior can be explained by the thicker tails of the Heston model which delivers more extreme market scenarios than the Black-Scholes model. Thus, in a more realistic financial market setting with stochastic volatility, the sub-optimality of the linear sharing rule is intensified.

\section{Conclusion}

In this article, we solve a collective investment problem of a fund manager who invests for a collective of individuals who measure their utility from the terminal wealth exceeding a deterministic minimum guarantee, both in a Black-Scholes model and a Heston model. We have shown that all the investors in the collective receive their individually optimal terminal wealth levels under financial fairness when the fund manager uses a specific state-dependent sharing rule. Using a financially fair linear sharing rule leads to moderate losses for all investors in the collective. However, imposing a linear sharing rule which is not financially fair makes some individuals better and some worse off, compared to the financially fair linear sharing rule. As ignoring the financial fairness condition can lead to drastic losses for some individuals, a financially fair linear sharing rule performs better from a fund manager's perspective if she wants to take account of all individuals in the collective in a fair way. Our results show that losses imposed by linear sharing rules are larger under stochastic volatility than under constant volatility.

It would be interesting to analyze how individual utility is affected if the fund manager is restricted to some commonly applied investment strategies like, for example, CPPI strategies. We leave this analysis for future research.

Acknowledgements Open Access funding enabled and organized by Projekt DEAL. Manuel Rach acknowledges the financial support given by the DFG for the research project "Zielrente: die Lösung zur alternden Gesellschaft in Deutschland" (Grant number 418318744). Furthermore, the authors thank anonymous referees for helpful comments and suggestions.

\section{Compliance with ethical standards}

Conflict of interest The authors declare that they have no conflict of interest.

Open Access This article is licensed under a Creative Commons Attribution 4.0 International License, which permits use, sharing, adaptation, distribution and reproduction in any medium or format, as long as you give appropriate credit to the original author(s) and the source, provide a link to the Creative Commons licence, and indicate if changes were made. The images or other third party material in this article are included in the article's Creative Commons licence, unless indicated otherwise in a credit line to the material. If material is not included in the article's Creative Commons licence and your intended use is not permitted by statutory regulation or exceeds the permitted use, you will need to obtain permission directly from the copyright holder. To view a copy of this licence, visit http://creativecommons.org/licenses/by/4.0/. 


\section{A Proof of Lemma 2}

To compute the conditional expectation in (20), let us additionally introduce the following notation:

$$
\begin{aligned}
Z_{t}^{+} & :=\rho W_{t}^{(1)}+\sqrt{1-\rho^{2}} W_{t}^{(2)}, \\
Z_{t}^{-} & :=\sqrt{1-\rho^{2}} W_{t}^{(1)}-\rho W_{t}^{(2)}, \\
\zeta_{t}^{ \pm} & :=\exp \left(-\eta_{ \pm} \int_{0}^{t} \sqrt{V_{s}} d Z_{s}^{ \pm}-\frac{\eta_{ \pm}^{2}}{2} \int_{0}^{t} V_{s} d s\right) .
\end{aligned}
$$

Note that $Z_{t}^{+}$and $Z_{t}^{-}$are independent Brownian motions because their covariation is equal to zero. Under this notation, we have

$$
\int_{s}^{t} \sqrt{V_{\nu}} d Z_{v}^{+}=\frac{1}{\delta}\left(V_{t}-V_{s}-\kappa \bar{V}(t-s)+\kappa \int_{s}^{t} V_{\nu} d v\right)
$$

and we can write $\xi_{t}^{(\eta)}=e^{-r t} \zeta_{t}^{+} \zeta_{t}^{-}$. This leads us to

$$
\mathbb{E}\left[\left(\frac{\xi_{T}^{(\eta)}}{\xi_{t}^{(\eta)}}\right)^{q_{i}} \mid \mathcal{F}_{t}\right]=e^{-r q_{i}(T-t)} \mathbb{E}\left[\left(\frac{\zeta_{T}^{+} \zeta_{T}^{-}}{\zeta_{t}^{+} \zeta_{t}^{-}}\right)^{q_{i}} \mid \mathcal{F}_{t}\right] .
$$

Conditioning on the path $\left\{Z_{s}^{+}\right\}_{s \in[t, T]}$, which is the Brownian motion driving the volatility, the process $\zeta_{t}^{-}$follows a log-normal distribution. Hence, using (44), this term can be expressed as

$$
\begin{gathered}
e^{-r q_{i}(T-t)} \mathbb{E}\left[\left(\frac{\zeta_{T}^{+}}{\zeta_{t}^{+}}\right)^{q_{i}} \mathbb{E}\left[\left(\frac{\zeta_{T}^{-}}{\zeta_{t}^{-}}\right)^{q_{i}} \mid\left\{Z_{s}^{+}\right\}_{s \in[t, T]}\right] \mid \mathcal{F}_{t}\right] \\
=e^{-r q_{i}(T-t)} \mathbb{E}\left[\left(\frac{\zeta_{T}^{+}}{\zeta_{t}^{+}}\right)^{q_{i}} e^{-q_{i}\left(1-q_{i}\right) \frac{\eta_{-}^{2}}{2} \int_{t}^{T} V_{s} d s} \mid \mathcal{F}_{t}\right] \\
=e^{-r q_{i}(T-t)} \mathbb{E}\left[e^{\left(V_{t}+\kappa \bar{V}(T-t)\right) a_{i}} e^{-a_{i} V_{T}-b_{i} \int_{t}^{T} V_{s} d s} \mid \mathcal{F}_{t}\right] \\
=e^{-r q_{i}(T-t)} e^{\left(V_{t}+\kappa \bar{V}(T-t)\right) a_{i}} \mathbb{E}\left[e^{-a_{i} V_{T}-b_{i} \int_{t}^{T} V_{s} d s} \mid \mathcal{F}_{t}\right] .
\end{gathered}
$$

The expectation in (45) is the Laplace transform of $\left(V_{T}, \int_{t}^{T} V_{s} d s\right)$ at $\left(a_{i}, b_{i}\right)$. An explicit formula for this Laplace transform and the necessary conditions for this representation are given in Proposition 5.1 in Kraft (2005) and Proposition 6.3.4.1 in Jeanblanc et al. (2009). It is shown in Chen et al. (2018) that assumption (19) is sufficient for the Laplace transform to be well-defined at $\left(a_{i}, b_{i}\right)$ for all $i=1, \ldots, n$. Therefore, we can simplify (45) to (20).

\section{References}

Andersen, T. G., Benzoni, L., \& Lund, J. (2002). An empirical investigation of continuous-time equity return models. The Journal of Finance, 57(3), 1239-1284.

Bakshi, G., \& Kapadia, N. (2003). Delta-hedged gains and the negative market volatility risk premium. The Review of Financial Studies, 16(2), 527-566.

Balder, S., \& Mahayni, A. (2010). How good are portfolio insurance strategies? In R. Kiesel, M. Scherer, \& R. Zagst (Eds.), Alternative Investments and Strategies (pp. 227-257). London: World Scientific Publishing Co., Ltd. 
Basak, S. (2002). A comparative study of portfolio insurance. Journal of Economic Dynamics and Control, 26(7-8), 1217-1241.

Benzoni, L. (1998). Pricing options under stochastic volatility: An econometric analysis. Manuscript, University of Minnesota.

Black, F., \& Jones, R. W. (1987). Simplifying portfolio insurance. The Journal of Portfolio Management, 14(1), 48-51.

Black, F., \& Perold, A. (1992). Theory of constant proportion portfolio insurance. Journal of Economic Dynamics and Control, 16(3-4), 403-426.

Branger, N., Chen, A., Gatzert, N., and Mahayni, A. (2018a). Optimal investments under linear sharing rules. Working paper. Available from the authors upon request.

Branger, N., Chen, A., Mahayni, A., and Nguyen, T. (2018b). Optimal collective investment. Working paper. Available at https://www.researchgate.net/publication/324910837.

Branger, N., Muck, M., Seifried, F. T., \& Weisheit, S. (2017). Optimal portfolios when variances and covariances can jump. Journal of Economic Dynamics and Control, 85, 59-89.

Branger, N., Schlag, C., \& Schneider, E. (2008). Optimal portfolios when volatility can jump. Journal of Banking \& Finance, 32(6), 1087-1097.

Bühlmann, H., \& Jewell, W. S. (1979). Optimal risk exchanges. ASTIN Bulletin: The Journal of the IAA, 10(3), 243-262.

Chacko, G., \& Viceira, L. M. (2005). Dynamic consumption and portfolio choice with stochastic volatility in incomplete markets. The Review of Financial Studies, 18(4), 1369-1402.

Chen, A., Nguyen, T., \& Rach, M. (2021). Optimal collective investment: The impact of sharing rules, management fees and guarantees. Journal of Banking \& Finance, 123, 106012.

Chen, A., Nguyen, T., \& Stadje, M. (2018). Optimal investment under VaR-regulation and minimum insurance. Insurance: Mathematics \& Economics, 79, 194-209.

Chernov, M., \& Ghysels, E. (2000). A study towards a unified approach to the joint estimation of objective and risk neutral measures for the purpose of options valuation. Journal of Financial Economics, 56(3), 407-458.

Cont, R., \& Tankov, P. (2004). Financial modelling with jump processes. Boca Raton, Florida: Chapman \& Hall/CRC.

Cox, J. C., \& Huang, C.-F. (1989). Optimal consumption and portfolio policies when asset prices follow a diffusion process. Journal of Economic Theory, 49(1), 33-83.

Cox, J., Ingersoll, J. E., Jr., \& Ross, S. A. (1985). A theory of the term structure of interest rates. Econometrica, 53(2), 385-408.

Dumas, B. (1989). Two-person dynamic equilibrium in the capital market. The Review of Financial Studies, 2(2), 157-188.

Eraker, B., Johannes, M., \& Polson, N. (2003). The impact of jumps in volatility and returns. The Journal of Finance, 58(3), 1269-1300.

Escobar, M., Ferrando, S., \& Rubtsov, A. (2018). Dynamic derivative strategies with stochastic interest rates and model uncertainty. Journal of Economic Dynamics and Control, 86, 49-71.

Fleming, W. H., \& Hernández-Hernández, D. (2003). An optimal consumption model with stochastic volatility. Finance and Stochastics, 7(2), 245-262.

Hambardzumyan, H., \& Korn, R. (2019). Dynamic hybrid products with guarantees-An optimal portfolio framework. Insurance: Mathematics and Economics, 84, 54-66.

Heston, S. L. (1993). A closed-form solution for options with stochastic volatility with applications to bond and currency options. The Review of Financial Studies, 6(2), 327-343.

Huang, C.-F., \& Litzenberger, R. (1985). On the necessary condition for linear sharing and separation: A note. Journal of Financial and Quantitative Analysis, 20(3), 381-384.

Jeanblanc, M., Yor, M., \& Chesney, M. (2009). Mathematical methods for financial markets. London: Springer.

Jensen, B. A., \& Nielsen, J. A. (2016). How suboptimal are linear sharing rules? Annals of Finance, 12(2), 221-243.

Jensen, B. A., \& Sørensen, C. (2001). Paying for minimum interest rate guarantees: Who should compensate who? European Financial Management, 7(2), 183-211.

Karatzas, I., Lehoczky, J. P., \& Shreve, S. E. (1990). Existence and uniqueness of multi-agent equilibrium in a stochastic, dynamic consumption/investment model. Mathematics of Operations Research, 15(1), 80-128.

Kim, H. H., Maurer, R., \& Mitchell, O. S. (2016). Time is money: Rational life cycle inertia and the delegation of investment management. Journal of Financial Economics, 121(2), 427-447.

Kraft, H. (2005). Optimal portfolios and heston's stochastic volatility model: an explicit solution for power utility. Quantitative Finance, 5(3), 303-313.

Liu, J. (2006). Portfolio selection in stochastic environments. The Review of Financial Studies, 20(1), 1-39. 
Liu, J., \& Pan, J. (2003). Dynamic derivative strategies. Journal of Financial Economics, 69(3), 401-430.

Merton, R. C. (1971). Optimum consumption and portfolio rules in a continuous-time model. Journal of Economic Theory, 3(4), 373-413.

OECD (2018). OECD Pensions Outlook 2018. OECD Pensions Outlook, OECD Publishing, Paris. Available at https://doi.org/10.1787/pens_outlook-2018-en.

Pain, D., \& Rand, J. (2008). Recent developments in portfolio insurance. Bank of England. Quarterly Bulletin, $48(1), 37-46$.

Pan, J. (2002). The jump-risk premia implicit in options: Evidence from an integrated time-series study. Journal of Financial Economics, 63(1), 3-50.

Pazdera, J., Schumacher, J. M., \& Werker, B. J. (2016). Cooperative investment in incomplete markets under financial fairness. Insurance: Mathematics and Economics, 71, 394-406.

Pham, H. (2002). Smooth solutions to optimal investment models with stochastic volatilities and portfolio constraints. Applied Mathematics \& Optimization, 46(1), 55-78.

Schumacher, J. M. (2018). Linear versus nonlinear allocation rules in risk sharing under financial fairness. ASTIN Bulletin: The Journal of the IAA, 48(3), 995-1024.

Temocin, B. Z., Korn, R., \& Selcuk-Kestel, A. S. (2018). Constant proportion portfolio insurance in defined contribution pension plan management. Annals of Operations Research, 266(1-2), 329-348.

Turner, J. A. (2014). Hybrid pensions: risk sharing arrangements for pension plan sponsors and participants. Society of Actuaries.

Weinbaum, D. (2009). Investor heterogeneity, asset pricing and volatility dynamics. Journal of Economic Dynamics and Control, 33(7), 1379-1397.

Wilson, R. (1968). The theory of syndicates. Econometrica: Journal of the Econometric Society, 36(1), 119132.

Xia, J. (2004). Multi-agent investment in incomplete markets. Finance and Stochastics, 8(2), 241-259.

Zieling, D., Mahayni, A., \& Balder, S. (2014). Performance evaluation of optimized portfolio insurance strategies. Journal of Banking \& Finance, 43, 212-225.

Publisher's Note Springer Nature remains neutral with regard to jurisdictional claims in published maps and institutional affiliations. 